\title{
Norma y precio del suelo urbano en la ciudad de Bogotá
}

\author{
Alex Araque. Universidad Sergio Arboleda, Bogotá, Colombia. \\ Lina Cantor. Universidad Sergio Arboleda, Bogotá, Colombia. \\ Paloma Moreno. Universidad Sergio Arboleda, Bogotá, Colombia.
}

RESUMEN | Este documento aborda la relación precios del suelo y norma urbana. El control urbano constituye el reconocimiento social en el territorio, las interacciones de las diversas clases sociales en la ocupación del suelo y su reflejo adecuado en los mercados. La teoría de la Renta Ofertada explica la configuración urbana a través de la demanda, la renta y los precios del suelo; la norma solo legitima la apropiación del excedente social. La convergencia norma y sociedad modula el precio del suelo urbano, dando fuerza a la norma. La estructura de rentas en Bogotá responde a los postulados de la Renta Ofertada en tanto lleva implícitas las desigualdades sociales y la baja capacidad de la administración de la ciudad para controlar las fuerzas del mercado. El resultado empírico señala que la norma no tiene efecto sobre los precios del suelo; es decir, el mercado domina la planificación de la ciudad.

PALABRas CLAVE $\mid$ mercado de suelo, gestión territorial, planificación urbana.

ABSTRACT | This document addresses the relationship between land prices and land regulations. Urban control constitutes the social recognition in the territory, the interactions of the different social classes in the occupation of the land, and its adequate reflection in the markets. The Bid Rent theory explains the urban configuration through demand, rent, and land prices; the norm only legitimizes the appropriation of the surplus. The convergence of norm and society modulates the cost of urban land, which gives strength to the norm. The structure of rents in Bogota responds to the postulates of Bid Rent in a way that implies social inequalities and the low capacity of the city's administration to control market forces. The empirical result indicates that the urban norm does not affect land prices; that is, the market dominates city planning.

KEYWORDS | land market, territorial management, urban planning.

Recibido el 16 de enero de 2020, aprobado el 24 de mayo de 2020.

E-mails: A. Araque, alex.araque@usa.edu.co | L. Cantor, lina.cantor@usa.edu.co | P. Moreno, palomamoreno07@gmail.com 


\section{Introducción}

La teoría de la renta del suelo -tanto urbana como rural- señala una causalidad unívoca entre presiones de demanda, renta y precios del suelo. En las presiones de demanda y el incremento del costo marginal de los bienes se originan las rentas del suelo en virtud de la utilización de técnicas productivas de beneficio nulo que expresan la irreproductibilidad del suelo. En el caso urbano esta relación unidireccional debe ser aún más clara, por cuanto bajo condiciones eminentemente teóricas el mercado configura la ciudad. En este sentido, la administración pública tiene un papel absolutamente servil del mercado: la norma legitima su mandato. El control urbano, entendido como la capacidad de la administración para orientar el territorio hacia un curso deseado, debe dirigir las fuerzas del mercado, dado que el bienestar colectivo no necesariamente se iguala a las necesidades del mercado. Una sociedad con instituciones sólidas seguirá las pautas de comportamiento establecidas por estas. Los predios rurales de la frontera urbana tendrán precios de aprovechamientos rurales sin incorporar expectativas de urbanización, por cuanto los propietarios creen en las instituciones y el control urbano derivado de esa fortaleza. En ese sentido, la norma bajo control urbano, entendido así, elimina las externalidades negativas del mercado. La credibilidad de los agentes en las autoridades urbanas impide usos de mercado no autorizados, la erradicación de la producción agrícola en la ruralidad de las grandes ciudades, y permite la ocupación del suelo por donde la sociedad lo determine. En este documento se muestra la inoperancia de la norma en Bogotá frente al direccionamiento del mercado, además de cómo las presiones del mercado determinan la evolución de los precios, tal como lo señala la teoría. La estructura del documento responde, en primer lugar, al papel de la norma. Posteriormente se detiene en la renta del suelo, poniendo énfasis en el origen de esta desde las presiones de la demanda. En la sección tercera se aborda la evidencia empírica, y en la sección final se presentan los aspectos centrales de la discusión y las conclusiones.

\section{La norma urbana}

El territorio es un hecho complejo. Es la suma del territorio natural, el construido, el organizado como referente de la existencia de una comunidad que se autorreconoce en ese territorio, el cual, a su vez, se encuentra regulado por un mecanismo político administrativo (Boisier, 2009; Brower, 1980). El territorio es un agente de transformación social y no debe ser considerado solo como un espacio funcional o un mero soporte físico de los objetos, actividades y procesos económicos (Alburquerque, 2006; Cuervo-González, 1998; Fundación DemucA, 2009; Jacobs, 1969; Naclerio et al., 2010). La norma urbana debe reflejar, entonces, esta complejidad, para concretar la cosmovisión social. No obstante, el dominio del planeamiento urbano en la práctica urbanística la dejó simplemente en prescripciones espaciales y arquitectónicas, en la regulación de sistemas urbanos cuyo gran producto de política pública es un plan físico, o unos códigos normativos, generalmente distantes de la economía política urbana y regional (Huxley, 1997; McLoughlin, 1994; Yiftachel, 1997). 
Por tradición, la práctica de la planificación urbana produce un conjunto de regulaciones sobre los usos y las intensidades de uso en el territorio, generalmente en el marco de planes de zonificación, además de definir estándares de construcción y un régimen de aprovechamientos y obligaciones urbanísticas, en ocasiones en el marco de planes maestros, planes físicos de diseño urbano y esquemas de construcción de infraestructuras urbanas. McLoughlin (1994) señala que la planificación urbana se convirtió en un debate sobre planes y regímenes regulatorios en lugar de lo que se suponía serían las mejores ciudades, poniendo gran énfasis no en lo que realmente sucede en el entorno construido -dinámicas sociales, económicas y políticas-, sino en los planes y el proceso de producción de esos planes. Sin embargo, la economía política espacial (o los estudios urbano-regionales) centra la discusión en la producción social del espacio; es decir, se cuestiona cómo funcionan realmente los lugares, las ciudades y las regiones, insistiendo en el papel fundamental de la geografía en la (re)conformación constante de las relaciones sociales (McLoughlin, 1994).

La forma urbana contribuye a la clasificación de la estructura espacial urbana, donde se distinguen los espacios adaptados, los flujos de personas y bienes y los patrones de interacción entre diferentes localizaciones (Chapin \& Hightower, 1965). Los estudios sobre el uso del suelo y el transporte señalan que prever más allá de unos diez años es técnica y políticamente muy difícil. En tanto, las alternativas de forma espacial no revelan las principales variables sociales que son de interés para la mayoría de las personas; por ejemplo, clase, raza, externalidades negativas, precios de la vivienda, entre otras. En este sentido, el anacrónico planeamiento urbano coincide con dinámicas de mercado que están reflejando claramente el mejor entendimiento que tienen los promotores inmobiliarios respecto de las preferencias individuales. Los planes maestros que rigen el planeamiento urbano muy a menudo son una función compleja de los promotores inmobiliarios, los especuladores de la tierra y los juegos de poder de los gobiernos locales (McLoughlin, 1994).

La gestión y la planificación del territorio desempeñan un papel fundamental en términos de promover el bienestar y el desarrollo local. En tal sentido, la norma urbana debe ser el resultado de los intereses colectivos y de la interpretación de las dinámicas sociales más allá de la eficiencia del mercado (Fischel, 2000; Wallace, 1988). Los elementos físicos que estructuran la ciudad derivan del urbanismo y la arquitectura, y sobre ellos se define el aprovechamiento del suelo (Vigliocco \& Meda, 1991). Los aprovechamientos, definidos en la norma, moderan el interés individual. No obstante, la geografía humana se proyecta como la ciencia que puede superar las tradicionales concepciones unidimensionales promercado de la ciudad, para alcanzar los mejores resultados de las intervenciones urbanas (Alonso, 1964; Burgess, 1925; Dear \& Flusty, 2002; Delgado \& Galindo, 2006; Harris \& Ullman, 1945; Hoyt, 1933). Es necesario recurrir a la geografía humana si se quiere avanzar hacia el centro de la economía política espacial en la discusión de la condición urbana (McLoughlin, 1994).

Ahora bien, tanto el planeamiento urbano como la economía política espacial requieren de control urbano. Frente al planeamiento urbano, el control es imperativo respecto de la imposición de regulaciones sobre la producción de la forma construida; en materia de economía política, el control urbano se refiere 
a la definición de normas con la colectividad y su debida apropiación, es decir, allí donde los habitantes se reconocen en la realidad social creada activamente por ellos (Martínez, 2014). En esta perspectiva, el control urbano hace referencia al seguimiento, la regulación, inspección, fiscalización e intervención sobre el sistema urbano, entendiendo este último como el conjunto de componentes dinámicos que conforman una ciudad, sus interrelaciones, y las actividades económicas, sociales y ambientales que se desarrollan en ella (Salazar \& Vergel, 2009, p. 3). Lo relevante al respecto es la legitimidad y credibilidad de la autoridad de la gestión urbanística y de los objetivos colectivos (Server, 1996). La carencia de control urbano implica la pérdida de la memoria colectiva, la pérdida del sentido de identificación del ciudadano con el medio urbano, el surgimiento de usos que modifican la dinámica espacial, la saturación excesiva de los bienes y servicios públicos, la pérdida de valores patrimoniales, entre otros (Luengo, 2002). En otras palabras, el asalto del mercado (Henderson \& Becker, 2000).

Al tener control urbano, la ciudad direcciona las fuerzas del mercado de manera que la norma tendría una estrecha relación con las dinámicas sociales y de allí con los precios del suelo (Jaramillo, 2009; Pollakowski \& Wachter, 1990; Thorson, 1994, 1997). La norma de aprovechamientos del suelo no genera renta del suelo en sí misma; solo dirige el mercado y de allí se capitaliza la renta del suelo una vez se concreta tal aprovechamiento (Glaeser \& Gyourko, 2002). Por ejemplo, las expectativas de incorporación de suelos rurales al perímetro de la ciudad inflan los precios de suelo rural al nivel de los de borde urbano. Los propietarios venderían a este precio, a un riesgo demasiado alto para el comprador sin la norma que legitime el uso urbano, o por el contrario esperan que la administración de la ciudad efectivamente incorpore ese suelo como suelo urbano y se concrete la renta urbana. La administración de la ciudad puede no incorporar a la ciudad predios rurales y dejarles el aprovechamiento agrícola por siempre, de tal manera que su precio será menor, equivalente al derivado de la renta agrícola. Esto último bajo el supuesto de credibilidad de los agentes en el control que ejerce la administración.

Una situación similar ocurre con la presión sobre los precios del suelo ante expectativas de cambios de usos al interior de la ciudad. En primer lugar, la renta del suelo ofertada por una localización es la primera fuerza que aparece en el mercado de una zona. En segundo lugar, esta oferta presiona al alza los precios de la propia zona, y por último presiona al alza los precios del suelo de zonas contiguas de usos distintos. La ciudad puede extirpar la presión al cambio de uso de las zonas contiguas incrementando la edificabilidad de las zonas altamente demandadas o estableciendo otras zonas de la ciudad en donde desarrollar estas actividades. En este caso legitima las presiones del mercado y permite la capitalización de la renta del suelo en las zonas de alta demanda, o dirige las fuerzas del mercado a otras zonas urbanas y de allí se genera renta del suelo en estas nuevas zonas. Es decir, cuando la administración de la ciudad tiene verdadero control urbano, decide la generación de las rentas del suelo con la norma. De otra parte, la invasión de aprovechamientos no permitidos en las zonas contiguas señala con claridad el bajo control de la ciudad sobre el mercado del suelo. 
Ahora bien, la teoría acerca de la influencia de la norma sobre los precios del suelo sugiere cierta endogeneidad entre ellas (Thorson, 1994). Esta es una confusión originada en la práctica avaluatoria que determina precios del suelo, pero no establece causalidad, solo la asume. En el caso de la técnica residual se parte de una norma, unos aprovechamientos asociados en edificación, y se calcula el precio del suelo como un residuo. Es evidente que el precio del suelo se determina en función del aprovechamiento edificatorio; de allí la conclusión de la causalidad norma y precio del suelo. No obstante, eso no tiene sentido en términos económicos. No es extraño ver en nuestras ciudades terrenos con aprovechamientos no validados por el mercado.

Sin control urbano, la norma simplemente reconoce las fuerzas del mercado (Maser et al., 1977). La ocupación de suelos por usos no permitidos es la regla y con ello solo les queda a las administraciones públicas, al expedir los actos administrativos, su legalización. Un caso dramático ocurrió con los humedales de Bogotá que fueron urbanizados. Entre 1950 y 1990, la ciudad perdió el $84 \%$ de estos cuerpos de agua (Aparicio et al., 2018; Cruz et al., 2017), y el Decreto 619 de 2000, Plan de Ordenamiento Territorial de Bogotá, nada dijo de su recuperación. De igual manera, la ocupación informal del suelo en varias ciudades es casi una tradición sobre la cual las ciudades desarrollan procesos de formalización (Camargo \& Hurtado, 2013; Fernandes, 2011).

La norma propone unos incentivos económicos, la administración genera las condiciones para que las dinámicas urbanas deseadas se produzcan (como la construcción de infraestructuras), pero el mercado es el ente que los valida (Monk et al., 1996; Yunda, 2019). Como ejemplo, en el Decreto 619 de 2000 se aprobó la expansión al sur de Bogotá en 1.195 hectáreas, lo que dio lugar al Decreto Distrital 252 de 2007, donde se aprobó el Plan de Ordenamiento Zonal de Usme. No obstante, en el Decreto 675 de 2018 se reconoce que, transcurridos más de diez años de la expedición del Decreto Distrital 252, no se ha podido ejecutar ninguna de las unidades de gestión urbanística allí propuestas. Justo en este panorama es donde se hace imperativo el uso de la economía política espacial en la planeación. Una administración urbana en línea con las dinámicas sociales establece un plan urbanístico legitimado por la colectividad y, en consecuencia, un mercado articulado, orientado por la sociedad. En este sentido, existe el planeamiento urbano sin control urbano. De nuevo, un entendimiento del territorio más allá de la anacrónica perspectiva del planeamiento urbano haría converger la política sobre el suelo con lo que se propone desde la economía política espacial (Huxley, 1997; Yiftachel, 1997).

\section{La renta del suelo}

Si bien la teoría de la renta del suelo de David Ricardo no tiene mucha utilidad para explicar los fenómenos urbanos, sí la tiene para establecer el mecanismo de funcionamiento de los precios y la formación de la renta del suelo en sus fundamentos. En general, como interesan los mecanismos diferenciales extensivo e intensivo y la formación del precio, se pasa rápidamente por aspectos que son cruciales. El principal de ellos es la necesidad del incremento del precio de los granos para pasar a un 
suelo de fertilidad inferior en el caso extensivo, o a una dosis adicional de capital en el caso intensivo. El precio debe financiar el costo de uso de los factores para usar una nueva técnica productiva.

En este sentido se debe señalar, primero, que la renta del suelo no hace parte de los precios de los bienes agrícolas en virtud de la formación del precio en el margen en donde el suelo, o la última inversión de capital y trabajo, no pagan renta. En segundo lugar, el paso de una técnica a otra exige que los precios de los granos hayan aumentado lo suficiente para financiar la remuneración de los factores de la nueva técnica, cuya producción es menor. Esto es absolutamente importante y debe ser resaltado: no se introduce una nueva técnica productiva si el precio no financia su costo. En tercer lugar, la direccionalidad de movimientos es inequívoca: altos precios del grano generan altas rentas del suelo. En conclusión, son las presiones de demanda las que generan alza en los precios de los bienes agrícolas y con este incremento aparecen las rentas del suelo.

Alonso (1964) sistematizó el mecanismo de la Renta Ofertada agrícola de Von Thünen en su versión urbana (Fujita \& Krugman, 1995). Esta teoría moderniza la teoría de la renta liberándola de las dificultades ricardianas, en particular de los rendimientos decrecientes. Se realiza un planteamiento del mercado del suelo desde la teoría neoclásica, en donde se incorporan los costos de transporte, dependientes de la distancia, y la disponibilidad a ofertar renta derivada del nivel de ingreso (Fujita, 1989). Dos aspectos se abordan en esta aproximación a la renta del suelo: el primero es la configuración de usos y, posteriormente, las presiones del mercado.

En el primer caso, Alonso (1964) conserva los supuestos de competencia perfecta y homogeneidad de suelo del modelo de Von Thünen. Se supone, adicionalmente, que el costo de oportunidad de la tierra es cero y cada actividad económica, industrial, residencial o comercial está dispuesta a pagar en función de la accesibilidad y cercanía al centro de la ciudad. Cada actividad localizada en el espacio configura un gradiente de renta ofertada (bid rent), que será definido por el excedente por unidad de tierra de cualquier productor de bienes $i$ en la ubicación $r$ (Fujita, 1989). La renta ofertada es la diferencia de los precios y los costos de transporte:

$$
\psi_{i}(r)=\left(p_{i}-t_{i} r\right) * q_{i}(r)
$$

Los beneficios estarán dados por la diferencia de los ingresos que perciba de su actividad, la renta ofertada en una situación de indiferencia frente a los costos de transporte por una localización $r$ y la renta del suelo de equilibrio. Es decir, todo beneficio positivo, derivado de una localización, será filtrado por el propietario del suelo, como lo señalan las siguientes ecuaciones:

$$
\begin{aligned}
& \pi_{i}(r)=\left(p_{i}-t_{i} r\right) * q_{i}(r)-R(r)=\psi_{i}(r)-R(r) \\
& R^{*}(r) \equiv \max \left\{\max \psi_{i}(r), 0\right\}
\end{aligned}
$$


en donde $R(r)$ es la renta por unidad de suelo prevaleciente en la distancia r. El beneficio obtenido por la actividad $i$ en $r$, en el margen es cero. Es decir, la renta ofertada es la renta de mercado. Nótese que en cada actividad económica se tienen infinitos productores cuyos márgenes son distintos, de manera que se configura un gradiente de rentas por cada una de las actividades urbanas, como se aprecia en la Figura 1.

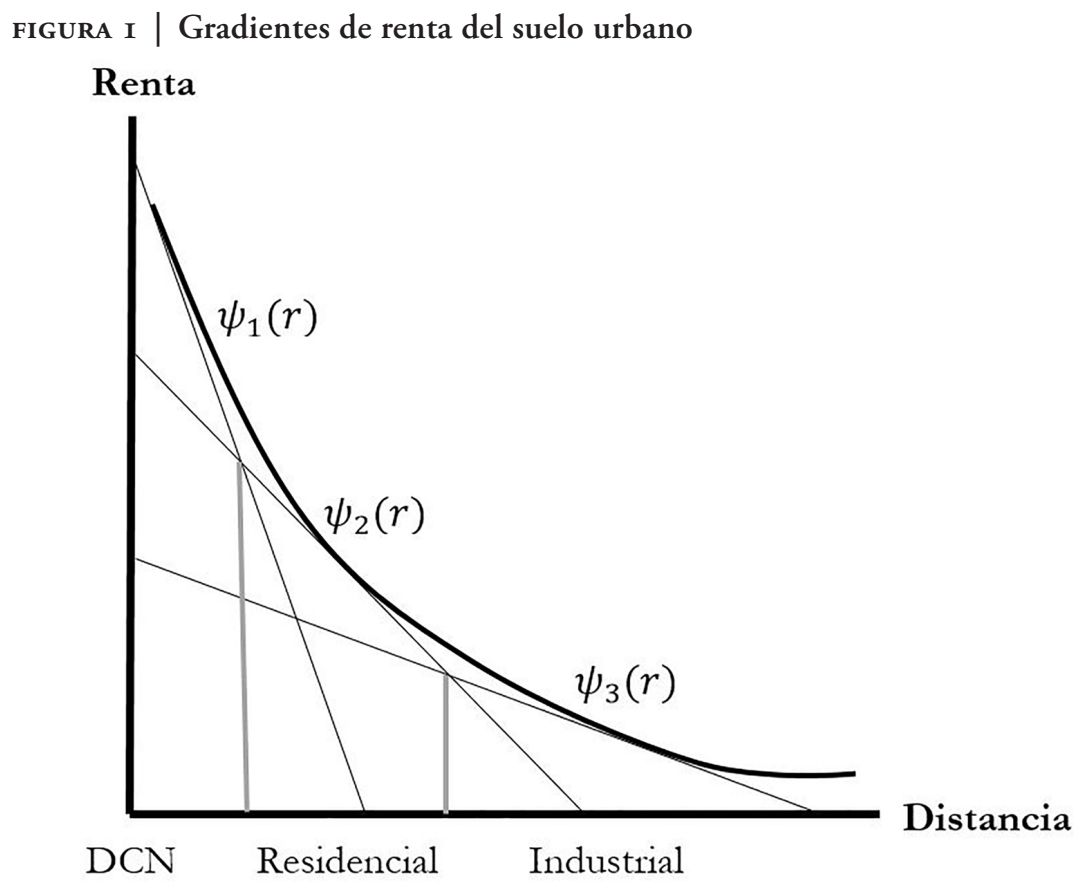

FUENTE: DISEÑO DE LOS AUTORES

De nuevo, en cada una de las localizaciones se presenta competencia entre productores por localización, de manera que solo se localizará allí el productor cuya oferta sea la superior, como lo indica la ecuación (3). Esta ecuación es la envolvente de rentas del suelo en este espacio. En este caso, en un esquema simple de ciudad monocéntrica, el mercado configura la ciudad en un Distrito Central de Negocios (DCN en adelante), una zona residencial, otra industrial, y la agrícola en las afueras de la ciudad. Los agentes descuentan los costos de producción en donde el de transporte es el central, como lo indica la ecuación (2). En este sentido, se supone un subastador que actúa en la ciudad, asignando rentas del suelo o costos de transporte. Para no pagar costos de transporte, cada agente ofrece la mayor renta del suelo posible sin salir del mercado. Es decir, el propietario del suelo drena cualquier beneficio positivo de una actividad económica. Igual ocurre en la actividad residencial: las diferencias de costos de transporte se equilibran con diferencias en el precio de la vivienda, por supuesto manteniendo constantes algunos atributos físicos. De esta manera, los hogares de altos ingresos pagan altas rentas para evadir altos costos de 
transporte, mientras que los hogares de bajos ingresos no tienen otra opción que recorrer largas distancias, por cuanto ofrecen una renta mucho menor.

\section{Las presiones de demanda}

El crecimiento poblacional/económico genera una mayor competencia y demanda por localización, como se aprecia en la Figura 2. Allí todos los gradientes y la envolvente se desplazan para representar la presión de demandas de localización sobre las rentas del suelo urbano. Las líneas punteadas seńalan un estado en el cual el mercado no es legitimado en usos por la administración de la ciudad. Las rentas del suelo han aumentado de manera notable en toda la ciudad, lo que se traduce en un indicador de modificación normativa.

FIGURA 2 | Gradientes de renta del suelo y presiones de mercado

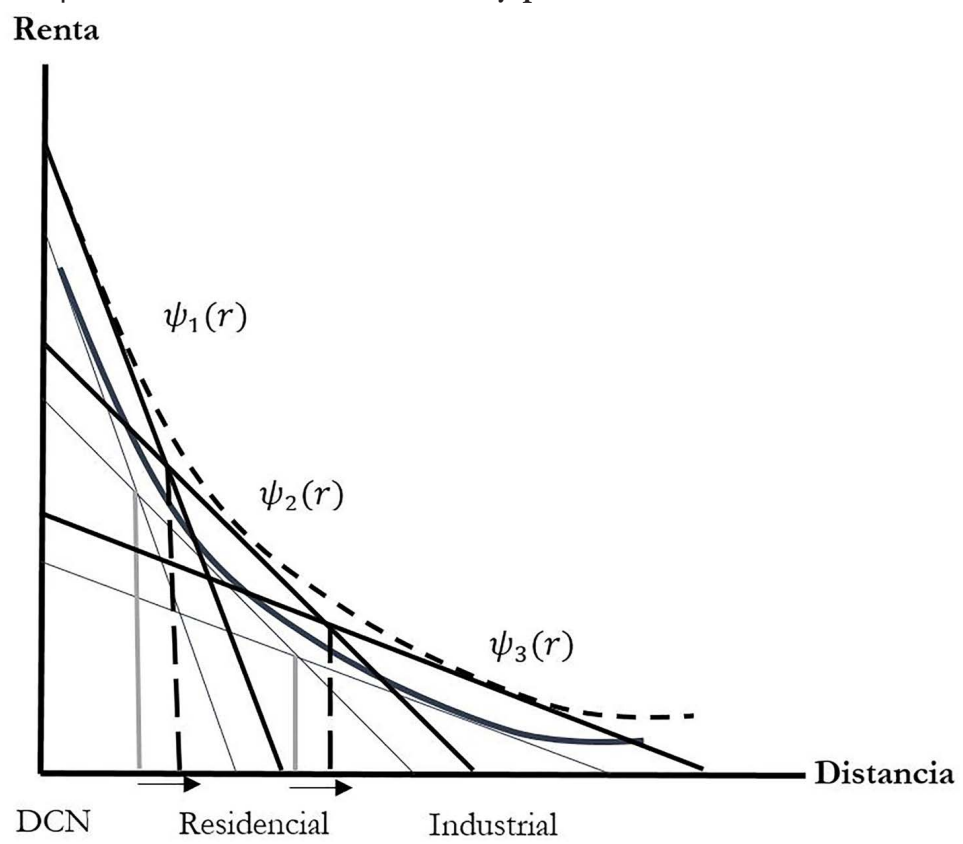

FUENTE: DISEÑO DE LOS AUTORES

En la Figura 2 se trazaron dos líneas verticales punteadas para señalar cómo en los bordes de las áreas de actividad se presiona al cambio de uso en las áreas adyacentes. Al final, una parte de las zonas residenciales recibe actividad comercial, las zonas industriales pierden áreas en favor de las residenciales, y las zonas rurales asumen los costos de las presiones del mercado en virtud de la desaparición de los cultivos en la frontera urbana. El punto central aquí es la irrelevancia de la norma frente a las presiones del mercado. En esta situación la norma no ha actuado, el mercado ha presionado los precios del suelo hacia arriba, lo que induce un cambio de usos del suelo; por tanto, no existe una rentabilidad superior en el uso antiguo que permita 
mantener la actividad en esa localización. La administración de la ciudad puede tomar al menos dos decisiones: legitimar las presiones del mercado mediante el cambio efectivo de usos del suelo o el incremento de las edificabilidades; o dirigir las fuerzas del mercado hacia los lugares que considere apropiados en función del ordenamiento urbano.

\section{Los múltiples centros}

El modelo monocéntrico requiere ampliarse para incorporar la compensación entre economías externas de escala en producción y deseconomías de escala en transporte, y con ello la existencia de varios centros en ciudades grandes (Helsley \& Sullivan, 1991). Los subcentros surgen cuando las deseconomías de transporte en el centro de la ciudad reducen el valor social de la mano de obra por los costos de transporte. De nuevo, esta es absolutamente una perspectiva económica de predominio del mercado. Bajo control urbano, la administración de la ciudad crea un subcentro sobre el cual dirige al mercado. No obstante, se reitera que a medida que la distancia al DCN o al Subcentro de Negocios (SN) aumenta, la renta del suelo, el precio de las viviendas, las alturas de los edificios y la densidad de población disminuyen (Brueckner, 2011).

\section{Evidencia empírica para Bogotá}

En esta sección se presentan los hechos ocurridos en Bogotá en el pasado reciente y largo plazo, que señalan la relación teoría, mercado y práctica urbanística. Los precios del suelo, el empleo y su dinámica de localización muestran cómo las presiones sobre la estructura urbana son los determinantes de los precios del suelo. La expansión urbana es igualmente la legalización de las presiones de mercado que conducen al crecimiento urbano. En este sentido, el planeamiento de Bogotá es irrelevante en la dirección de las fuerzas del mercado, salvando contadas excepciones, como Unicentro y Ciudad Salitre.

\section{Los precios del suelo}

En primer lugar, la ordenación de los precios del suelo y de la estructura urbana en Bogotá parecen replicar la configuración de las rentas del suelo señaladas en los textos (Fujita, 2010; Fujita \& Thisse, 2013), pero no la eficiencia de mercados autorregulados de Henderson y Becker (2000), si se consideran los altos costos de transporte derivados de la congestión en la ciudad. En la Figura 3 se presentan los precios del suelo por metro cuadrado de las zonas de la Lonja de Propiedad Raíz de Bogotá (LPRB), entidad gremial que agrupa a las personas naturales y jurídicas vinculadas al sector inmobiliario de la ciudad, cuya publicación sobre los precios del suelo data de 1960 . 


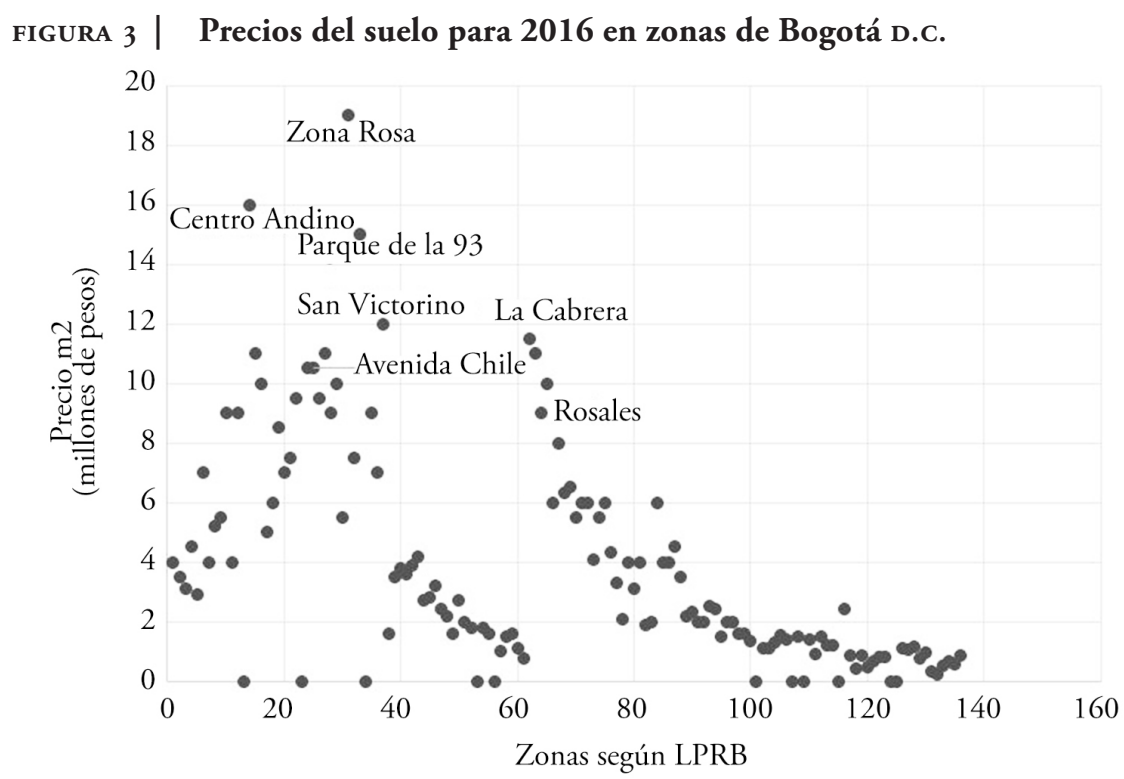

FUENTE: ELABORACIÓN PROPIA CON DATOS DE LPRB

En la Figura 3 se aprecian dos mundos separados: el comercial y el residencial. En el primer caso, la Zona Rosa, el Centro Andino, el Parque de la 93 y San Victorino, se consolidan como las zonas de mayor precio; y en la zona residencial, La Cabrera y Rosales constituyen el punto de precios más altos de Bogotá. La distancia horizontal está asociada a la numeración dada por la LPRB y no a su cercanía geográfica. En el área a la izquierda del número 20 en el eje horizontal de la Figura 3 se tienen las zonas comerciales tradicionales del Distrito Central de Negocios y las ampliadas hasta el Teleport Business (LPRB, 2017). En la Figura 3 se pueden correr las zonas para acercarse a la distancia geográfica y no habría diferencia con la relación preciodistancia de la envolvente de la ecuación (3).

Al observar la dinámica de precios de la ciudad, se puede sugerir que la configuración de la misma es determinada por el mercado, mientras el planeamiento urbano tiene menor fuerza en su configuración. El conteo de Zonas de la LPRB indica un crecimiento sostenido del mercado inmobiliario y la expansión del Distrito Central de negocios y de los centros complementarios. En otras palabras, mientras en 1960 la LPRB dividía la ciudad en 24 zonas para determinar los precios del suelo, para 2016 se tenían 125 zonas, lo que muestra cómo el mercado de suelo se ha acelerado en el tiempo; en particular, el incremento de 31 zonas entre 1987 y 1988 no tiene equivalente en nada anterior. Ese ańo, los avaluadores de la LPRB reconocieron la importancia de la expansión del centro tradicional hacia el norte por la Carrera 11 desde la Calle 73 a la Calle 100 y en la zona de Ciudad Salitre, un ejemplo de las presiones de precios originadas en las demandas de localización en la ciudad. 


\section{El empleo}

Un segundo hecho en que incide la relación teoría, mercado y práctica urbanística, es la localización del empleo. En los mapas de la Figura 4 aparecen las centralidades y las operaciones estratégicas del Decreto 190 de 2004. Esta figura muestra un proceso de consolidación, saturación y expansión de los centros de empleo en la ciudad. La actividad económica llena los intersticios dejados por las edificaciones residenciales, ocupa aquellos espacios y satura las periferias de los centros existentes, presionando su expansión. Dos ejemplos permiten asociar las presiones al cambio de uso: Quinta Paredes y La Castellana, sectores con origen residencial, pero donde las presiones de localización de hoteles, servicios financieros y servicios comerciales erradicaron el uso residencial en gran parte de ellos.

FIGURA 4 | Localización del empleo en Bogotá, 1990, 1994, 2001

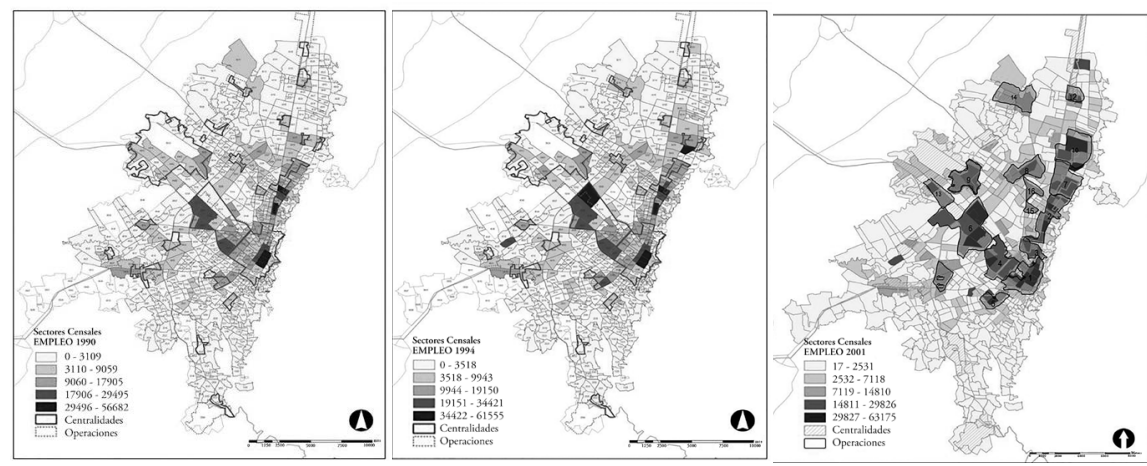

FUENTE: DEPARTAMENTO ADMINISTRATIVO NACIONAL DE ESTADÍSTICA (DANE): CENSOS DE POBLACIÓN Y VIVIENDA - CENSO ECONÓMICO NACIONAL Y MULTISECTORIAL. CÁLCULOS DE LOS AUTORES

\section{La expansión urbana}

El rápido y desbordado crecimiento poblacional de Bogotá generó una fuerte demanda de localización residencial y, con ella, económica. La respuesta de la administración fue permitir cambios y expansión de usos del suelo dentro del perímetro urbano y cambios en la clasificación del suelo para ofertar suelo a los hogares de bajos ingresos. Estas decisiones implicaron el desbordamiento de la ciudad sobre centros urbanos menores, como Usaquén, Suba, Engativá, Fontibón y Usme. Lo relevante de estas decisiones es su efecto sobre las expectativas del mercado de suelo rural en la frontera de las ciudades. Para tal efecto, se estimó por medio de Mínimos Cuadrados Ordinarios el logaritmo del precio del metro cuadrado de suelo rural (lps) en función del área del terreno (lat), de la distancia al perímetro urbano (ldist), del área construida (lac) y de los distintos usos del suelo $\left(U_{s o} o_{i}\right)$, como se señala en la siguiente ecuación:

$$
\text { lps }=\beta_{1}+\beta_{2} \text { lat }+\beta_{3} \text { ldist }+\beta_{4} \text { lac }+\beta_{5 i} U s o_{i}+\mu_{1}
$$


La variable uso es una variable factor que se estructura a partir de los usos del suelo: habitacional, institucional, comercial, industrial, los usos de perfil rural como enramadas, caballerizas, establos, entre otros, y finalmente los predios sin usos identificados. El término de perturbación estocástica se supone bien comportado. De la base de información se excluyeron los predios con áreas inferiores a 100 metros cuadrados y los de áreas superiores a 161.000 metros cuadrados clasificados como suelos de protección.

TABLA I Regresión de los precios del suelo rural

\begin{tabular}{|c|c|}
\hline VARIABLE & ESTIMADOR \\
\hline Logaritmo del área terreno & $\begin{array}{l}-0,5410154^{* * *} \\
(0,0114862)\end{array}$ \\
\hline Logaritmo de la distancia & $\begin{array}{l}-0,3378636^{* * *} \\
(0,0204127)\end{array}$ \\
\hline Logaritmo del área edificada & $\begin{array}{l}0,6331522^{* * *} \\
(0,0233492)\end{array}$ \\
\hline \multicolumn{2}{|l|}{ Uso (Residencial) } \\
\hline Institucional & $\begin{array}{l}-0,91702^{* * *} \\
(0,1711841)\end{array}$ \\
\hline Comerciales & $\begin{array}{l}0,1394812 \\
(0,1073896)\end{array}$ \\
\hline Industrial & $\begin{array}{l}-2,139326^{* * *} \\
(0,4777265)\end{array}$ \\
\hline Usos económicos de perfil rural & $\begin{array}{c}-0,3472471^{*} \\
(0,1620363)\end{array}$ \\
\hline Sin clasificar & $\begin{array}{l}-0,1529621 \\
(0,4184889)\end{array}$ \\
\hline Constante & $\begin{array}{l}13,22426^{* * *} \\
(0,2183833)\end{array}$ \\
\hline $\mathrm{N}$ & 4275 \\
\hline
\end{tabular}

FUENTE: ELABORACIÓN PROPIA

En la Tabla 1, los resultados del modelo indican que los predios de gran magnitud tienen precios menores. Esto es adecuado en virtud de que el loteamiento incrementa el precio del suelo. Respecto de la distancia, se tiene que, al alejarse del perímetro urbano en un $100 \%$, el precio por metro cuadrado se reduce en cerca de $33,7 \%$. De otra parte, si el predio tiene área construida, su precio se incrementará notablemente con áreas edificadas mayores. Para la variable uso, donde el residencial es la base de referencia para la estimación, todos los usos tienen menores precios respecto a los usos residenciales, excepto los comerciales y sin clasificar, que no son estadísticamente significativos. Los usos institucionales caen bastante, cerca del $92 \%$ frente al residencial; y los industriales, 213\%. Esto significa que si un predio institucional tiene un suelo cuyo precio es de $522 \mathrm{mil}$ pesos, el residencial 
será de 1 millón de pesos el metro cuadrado. En el caso de los usos industriales, el precio sería 318 mil pesos y el residencial, de 1 millón; cabe aclarar que en esta categoría se tienen cerca de 10 registros. Las actividades rurales, como las enramadas y caballerizas, tienen un precio superior a los anteriores e inferior solo en el 34,7\% al residencial de borde urbano. Este último resultado deja ver que la norma no ha impedido que el suelo rural contenga expectativas de incorporación al suelo urbano. De otra parte, en el pasado reciente, 1997-2016, las presiones sobre el suelo tuvieron la misma respuesta en los municipios que colindan con Bogotá y en un segundo anillo que conserva una relación funcional con la ciudad. En primer lugar, en la dinámica de ocupación sobre la región, la característica central es la existencia de espacios intermunicipales no ocupados. En segundo lugar, lo central es la predominancia de algunos corredores, como el de ZipaquiráMosquera-Fusagasugá; y, en tercer lugar, las dinámicas de las densidades describen las lógicas de las acciones de las administraciones públicas.

TABLA 2 Tasa de crecimiento poblacional y densidades (porcentajes)

\begin{tabular}{|c|c|c|c|}
\hline \multirow{2}{*}{ PERIODO } & BOGOTÁ & REGIÓN & 2O MUNICIPIOS \\
\cline { 2 - 4 } & \multicolumn{3}{|c|}{ POBLACIÓN (\%) } \\
\hline $1997-2016$ & 1,54 & 2,10 & 5,28 \\
\hline $2010-2016$ & 1,34 & 2,10 & 5,56 \\
\hline & \multicolumn{3}{|c|}{ DENSIDAD DE POBLACIÓN (\%) } \\
\hline $1997-2016$ & 0,80 & $-0,60$ & $-2,28$ \\
\hline $2010-2016$ & 1,17 & 0,21 & 1,20 \\
\hline
\end{tabular}

FUENTE: IDOM, SECRETARÍA DISTRITAL DE PLANEACIÓN (SDP), GOBERNACIÓN DE CUNDINAMARCA Y FINDETER (2OI8)

De acuerdo con la Tabla 2, la población de Bogotá en el periodo 1997-2016 creció de manera continua al 1,54\% anual, mientras que la de los veinte municipios lo hizo al impresionante ritmo del 5,28\% anual. En los últimos seis años del periodo, se aprecia una menor tasa de crecimiento poblacional en Bogotá; según el Departamento Administrativo Nacional de Estadística (DANE) (2019), la ciudad es expulsora de población, lo que se debe, por un lado, al aumento sostenido de los precios de la vivienda en Bogotá, que ha obligado a los hogares de ingreso medio bajo y bajo a buscar vivienda en las afueras de la ciudad; y por otro, está el desplazamiento de los hogares con ingresos altos a municipios que tienen una oferta de vivienda con gran cantidad de amenidades ambientales y un precio mejor del que podrían encontrar en la ciudad, como, por ejemplo, la vivienda campestre (Amézquita et al., 2015; IDOM et al., 2018). La densidad de población creció en Bogotá en ambos periodos, pero la de las otras regiones descendió. Esto indica que la acción de las administraciones de las ciudades de la región fue incrementar el perímetro urbano, a una tasa del 7,5\% anual, como modelo urbano. Es decir, se desarrolló un modelo de ciudad disperso, de manera que esta medida afectó todas las áreas rurales del sistema de ciudades y, con ello, los sistemas naturales. 


\section{La relación de precios}

Ahora bien, si la teoría funciona, los mercados deben comportase como ella lo señala. Es decir, la presión de demanda de localización es la fuente de crecimiento de los precios del suelo, de manera que deberían ocurrir al menos tres cosas. Primero, las tasas de crecimiento de los precios del suelo de zonas conexas deben ser similares o los precios deben estar cointegrados. En segundo lugar, como se explica en la Figura 2, con las presiones de precios del suelo laterales debería existir causalidad en el sentido de Granger de la zona dominante a la dominada, así como lo sugiere la teoría bajo economías de escala y la ciudad como una firma. Un incremento del salario hace que la envolvente de los gradientes de renta del suelo se desplace hacia arriba y la ciudad se expanda (Fujita \& Thisse, 2013). Es decir, los valores pasados de los precios de la zona residencial son afectados por los precios pasados de la zona comercial (Enders, 1995; Lütkepohl \& Krätzig, 2004; Madsen, 2007). Finalmente, los cambios normativos no deberían tener efecto-precio en virtud de la dinámica propia de los precios derivada de las presiones de localización (Maser et al., 1977; Pollakowski \& Wachter, 1990). Para probar estas hipótesis se trabaja con las series de la LPRB para la zona Avenida Chile, teniendo en cuenta que es zona comercialfinanciera y está dentro del DCN de Bogotá, y las zonas residenciales La Cabrera y Rosales, como aquellas asociadas y contiguas. La Tabla 3 presenta su evolución temporal en términos estadísticos.

TABLA 3 | Tasas de crecimiento zonas Avenida Chile, La Cabrera y Rosales

\begin{tabular}{|l|c|c|c|}
\hline \multicolumn{1}{|c|}{ ZONA } & AVENIDA CHILE & LA CABRERA & ROSALES \\
\hline Constante & $-133,2066^{* *}$ & $-43,82341$ & $-77,47145^{* *}$ \\
\hline Tasa de crecimiento 1960-2016 & $0,0743574^{* *}$ & $0,0286446^{* *}$ & $0,0456483^{* *}$ \\
\hline D_79 & 54,92455 & $-77,17567^{* *}$ & $-37,47788$ \\
\hline Break Point_79 & $-0,0277275$ & $0,0392709^{* *}$ & 0,0191112 \\
\hline R2_adj & 0,8883 & 0,9411 & 0,9339 \\
\hline
\end{tabular}

NOTA: ** ESTADÍSTICAMENTE SIGNificativo (AL 99\%)

FUENTE: ELABORACIÓN PROPIA

En la tabla, se tienen la constante, la tasa de crecimiento anual, el cambio en el intercepto derivado de un cambio en la pendiente en el periodo posterior a 1978 en las series (D_79; Break Point_79) y el R cuadrado ajustado para cada una de las zonas en análisis. En las restantes columnas se presentan los estimadores para cada zona. En primer lugar, los precios del suelo de Avenida Chile aumentan a una tasa del 7,4\%, mientras que las zonas residenciales en consideración, La Cabrera y Rosales, lo hacen al 2,8\% y 4,5\% anual, respectivamente. En segundo lugar, en La Cabrera el cambio estructural, BP_79, sí es estadísticamente significativo, mientras que en las otras zonas no lo es. Esto significa que, en el último periodo, 1978-2016, los precios de este sector crecieron al 6,8\% anual aproximadamente, dinámica cercana a la de la Avenida Chile. En general, las zonas residenciales tienen una tasa de incremento de los precios menor a la de la zona comercial-financiera. Como la correlación de las dos zonas residenciales es del 99\% y la tasa de crecimiento de La 
Cabrera es más cercana a la de la Avenida Chile, se tomará esta última para el análisis propuesto. El análisis se centra en los precios de La Cabrera en asociación con Avenida Chile. Para probar las hipótesis de irrelevancia de la norma en los precios del suelo se estimó la siguiente ecuación:

$$
l p c=\beta_{0}+\beta_{1} p i b+\beta_{2} l l c v+\beta_{3} a n t+\beta_{4} a d j+\beta_{5} l A v C h+\beta_{6} a u p z+\beta_{7} a d u p z+\mu
$$

en donde los precios de La Cabrera $(l p c)$ son función de la actividad económica (pib); de las licencias de edificación en vivienda ( $l l c v)$; de una variable de anticipación de la expedición del Plan de Ordenamiento Territorial (рот) (ant), que toma valores de 0,3 en el año 1998, de 0,6 en 1999, de 0,9 en el ańo 2000 y 1 en años posteriores; del ajuste a la norma del рот en general (adj), variable de escalamiento $0,25,-0,5,-0,75$ y 1 en un periodo de cuatro ańos; de los precios del centro económico más cercano, Avenida Chile $(l A v C h)$; y de la zonificación de la Unidad de Planeamiento Zonal (UPZ) en particular (aupz y adupz) expedida en el año 2010 para esa zona. En este último caso, el periodo de anticipación (aupz) y de ajuste (adupz) es solo de tres años. El término de perturbación sigue un proceso ARMa que captura los comportamientos de los propietarios en el pasado y su respuesta a shocks en este mercado:

$$
\mu_{t}=\sum_{i=1}^{n} \alpha_{i} \mu_{t-i}+\sum_{i=1}^{n} \gamma_{i} \varepsilon_{t-i}
$$

cuyo orden debe ser identificado en las estimaciones. En la Tabla 4 se presentan los resultados de la estimación del precio del suelo de La Cabrera, donde se incluyen el precio de la Avenida Chile y los componentes transitorios autorregresivos de orden 2. Solo el área licenciada en vivienda en Bogotá y los precios del suelo en la Avenida Chile son estadísticamente significativos.

De acuerdo con los resultados, un incremento del 10\% de los precios en la zona comercial-financiera Avenida Chile, genera un incremento del 8,86\% en la zona residencial La Cabrera. Un 10\% de incremento en las presiones de demanda de vivienda en la ciudad reduce los precios de La Cabrera en un 0,9\%. Para este resultado se puede proponer como hipótesis que la saturación de vivienda genera congestión y los que más costos asumen son los hogares de altos ingresos, los cuales buscan evadir la congestión vía la localización. Por su parte, el coeficiente del componente autorregresivo de primer orden indica un proceso especulativo en la formación de precios del suelo, y el coeficiente de segundo orden indica un ajuste que hace el mercado a esa presión al alza para regular una burbuja, pero su convergencia a los precios de equilibrio de largo plazo es lenta. La velocidad de convergencia es de 0,8942, como se puede apreciar en la suma de los coeficientes L1 y L2 de la Tabla 4; es decir, los promotores inmobiliarios compran el suelo y, si se presenta alguna crisis, no salen a vender a bajo precio, esperan sin angustia. Si bien esto es importante, lo es más la presión de precios de las zonas comerciales, que incrementa muy por encima la pérdida originada en la congestión. Aquí la norma tiene efecto nulo sobre los precios del suelo. 
TABla 4 Modelo ARIMA La Cabrera

\begin{tabular}{|c|c|c|}
\hline \multicolumn{3}{|c|}{ LOGARITMO DEL PRECIO DEL SUELO DE LA CABRERA } \\
\hline \multicolumn{2}{|l|}{ PIB } & $\begin{array}{c}0,173 \\
(0,214)\end{array}$ \\
\hline \multicolumn{2}{|c|}{ Licencias de construcción } & $\begin{array}{l}-0,090^{* *} \\
(0,034)\end{array}$ \\
\hline \multicolumn{2}{|c|}{ Efecto anticipación POT } & $\begin{array}{c}0,187 \\
(0,448)\end{array}$ \\
\hline \multicolumn{2}{|c|}{ Efecto ajuste POT } & $\begin{array}{c}0,316 \\
(0,464)\end{array}$ \\
\hline \multicolumn{2}{|c|}{ Precios del suelo Avenida Chile } & $\begin{array}{l}0,886^{* * *} \\
(0,080)\end{array}$ \\
\hline \multicolumn{2}{|c|}{ Efecto anticipación zonificación } & $\begin{array}{c}0,189 \\
(0,268)\end{array}$ \\
\hline \multicolumn{2}{|c|}{ Efecto ajuste zonificación } & $\begin{array}{l}-0,032 \\
(0,259)\end{array}$ \\
\hline \multicolumn{2}{|c|}{ Constante } & $\begin{array}{l}-0,601 \\
(3,177)\end{array}$ \\
\hline \multicolumn{3}{|c|}{ ARMA } \\
\hline \multicolumn{2}{|l|}{ L1 } & $\begin{array}{l}1,490^{* * *} \\
(0,081)\end{array}$ \\
\hline \multicolumn{2}{|l|}{$\mathrm{L} 2$} & $\begin{array}{l}-0,596^{* * *} \\
(0,095)\end{array}$ \\
\hline \multicolumn{2}{|l|}{$\mathrm{N}$} & 57 \\
\hline \multicolumn{3}{|c|}{ NOTA: $\quad$ ERRORES ESTÁNDAR EN PARÉNTESIS. ${ }^{*} \mathrm{P}<0,05,{ }^{* *} \mathrm{P}<0,0 \mathrm{I},{ }^{* * *} \mathrm{P}<0,000 \mathrm{I}$} \\
\hline \multicolumn{3}{|c|}{$\begin{array}{l}\text { ARIMA: ACRÓNIMO DEL INGLÉS AUTOREGRESSIVE INTEGRATED MOVING AVERAGE (MODELO AUTORREGRESIVO } \\
\text { INTEGRADO DE MEDIA MÓVIL) }\end{array}$} \\
\hline \multicolumn{3}{|r|}{ DELO AUTORREGRESIVO DE MEDIA } \\
\hline
\end{tabular}

En el caso de la Avenida Chile, se corrieron las especificaciones de las ecuaciones (5) y (6). Las variables estadísticamente significativas son el PIв y los componentes autorregresivos de orden 1 y 2 . Se puede apreciar que un incremento del $10 \%$ en el PIB genera un incremento de los precios de este suelo en $14,7 \%$. La suma de los coeficientes de los términos autorregresivos -es decir, la velocidad de convergencia al equilibrio de largo plazo $(0,724)$ - es menor que la de La Cabrera. Aquí el ajuste en el segundo periodo corresponde al del sexto periodo de La Cabrera. Las actividades comerciales necesitan un ajuste rápido, mientras que los agentes residenciales tienen un mayor tiempo de espera. Es importante resaltar que en los modelos presentados en la Tabla 4 y Tabla 5, las normas (UPZ у рот) tienen un efecto nulo sobre los precios del suelo. 
Tabla 5 | Modelo arima Avenida Chile

\begin{tabular}{|c|c|}
\hline \multicolumn{2}{|c|}{ LOGARITMO DEL PRECIO DEL SUELO DE LA AVENIDA CHILE } \\
\hline PIB & $\begin{array}{l}1,468^{* * *} \\
(0,353)\end{array}$ \\
\hline Licencias de construcción & $\begin{array}{c}0,017 \\
(0,162)\end{array}$ \\
\hline Diferencia de la serie del PIB & $\begin{array}{c}0,111 \\
(1,880)\end{array}$ \\
\hline Efecto anticipación РОТ & $\begin{array}{l}-0,324 \\
(0,581)\end{array}$ \\
\hline Efecto ajuste POT & $\begin{array}{l}-0,179 \\
(0,562)\end{array}$ \\
\hline Efecto anticipación zonificación & $\begin{array}{c}0,368 \\
(1,160)\end{array}$ \\
\hline Efecto ajuste zonificación & $\begin{array}{c}0,155 \\
(1,842) \\
\end{array}$ \\
\hline Constante & $\begin{array}{r}-11,743^{*} \\
(5,717) \\
\end{array}$ \\
\hline \multicolumn{2}{|l|}{ ARMA } \\
\hline L1 & $\begin{array}{l}1,018^{* * *} \\
(0,132)\end{array}$ \\
\hline $\mathrm{L} 2$ & $\begin{array}{l}-0,294^{*} \\
(0,128)\end{array}$ \\
\hline $\mathrm{N}$ & 56 \\
\hline
\end{tabular}

Pruebas de raíz unitaria, de cointegración y causalidad

Las pruebas de raíz unitaria indican que las series de precios no son estacionarias; es decir, su varianza depende del tiempo. Una prueba de cointegración indica que existe un vector cointegrante entre las dos series, lo que implica una relación de equilibrio entre las dos variables; dicha relación se puede salir del equilibrio, pero retorna por su estacionariedad derivada del vector cointegrante. En la Figura 5 se aprecia la evolución en crecimiento y retroceso de estos precios.

En la figura también se aprecia cómo en algunos momentos del tiempo, en la década de los sesenta, la asociación mostraba un crecimiento apenas notable en la Avenida Chile y una respuesta marginal en La Cabrera. En los ochenta, la dinámica de precios se acelera y comparten la misma evolución. Juntas crecen, la Avenida Chile un poco más, y en periodos de crisis marcados por depresiones económicas también retroceden. En el periodo final de la muestra, los precios han tenido un crecimiento sostenido, pero La Cabrera ha acelerado su crecimiento, lo que puede significar la expansión del centro a áreas mayores a las ya consideradas. Los resultados sugieren que los precios de la Avenida Chile causan los precios de La Cabrera en el sentido de Granger. No hay evidencia de causalidad de precios de La Cabrera a la Avenida Chile. Lo anterior permite argumentar que el comportamiento de los precios es como lo explica la teoría de la renta del suelo ofertada: presiones de demanda sobre la estructura urbana, alzas de precios en el DCN, que se expanden al resto de las zonas de la ciudad. 
FIgURA 5 | Logaritmo de precios La Cabrera y Avenida Chile

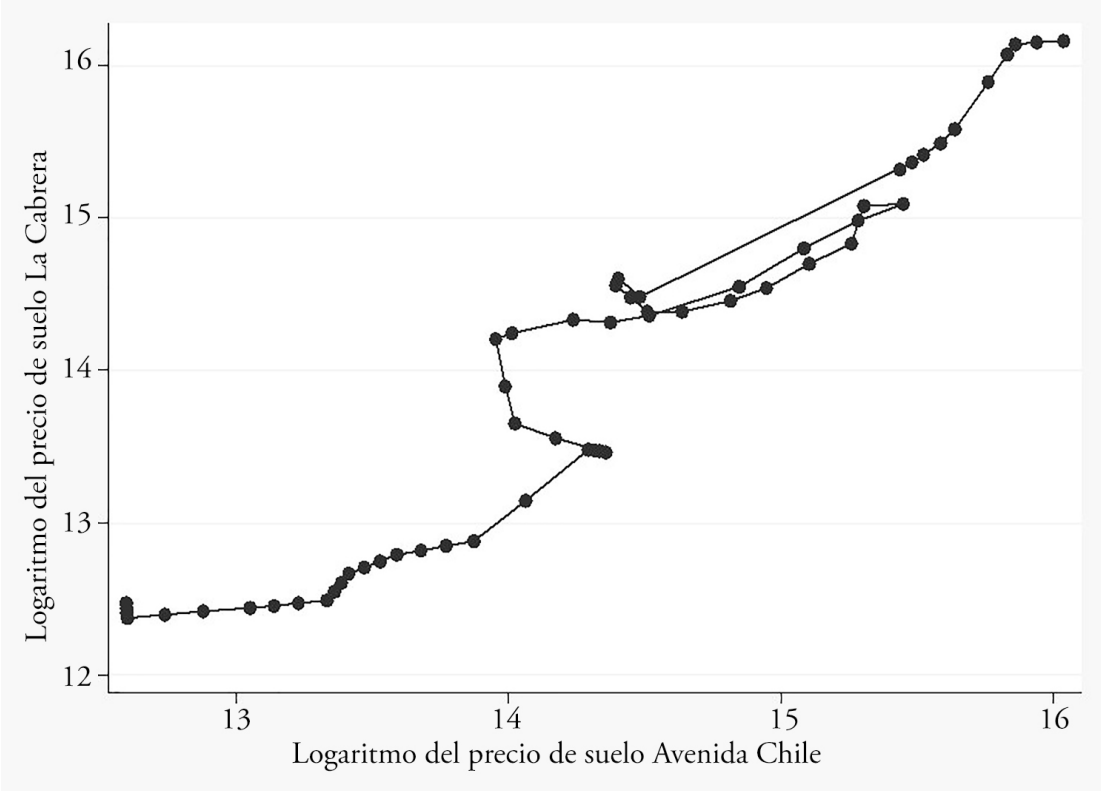

FUENTE: ELABORACIÓN PROPIA

\section{Discusión y conclusiones}

La identificación de la norma urbana con el resultado de los procesos de discusión de la sociedad sobre sus espacios de ocupación permite preguntarse por el entendimiento de la administración urbana sobre cómo esta se identifica con el territorio. Espacios urbanos donde la normativa de ocupación no se cumplió, usos comerciales no permitidos en zonas residenciales, ocupación y desarrollo informal indican un divorcio entre norma y sociedad y un llamado urgente a su convergencia. Tal como se entiende en esta investigación, el control urbano se alcanza mediante la armonización de la norma con el bienestar de la sociedad, de manera de superar con ello las meras prescripciones espaciales y la regulación mediante códigos normativos asociada al planeamiento urbano tradicional.

La relación entre la norma y precios del suelo en la economía espacial debe ser clara. En primer lugar, las presiones de localización incrementan la oferta de renta del suelo y, por tanto, su precio. Es decir, la demanda por el suelo es la fuerza originaria que presiona los precios de los bienes, y de allí se genera la renta del suelo y se concreta en el precio. La norma tiene la capacidad de orientar y dirigir las fuerzas del mercado y, por tanto, las demandas de localización deben concretarse de manera conveniente para el bienestar colectivo. La desarticulación entre la norma y la sociedad se evidencia en la irrelevancia de la norma en la definición de los precios del suelo, tal como se mostró en este documento. 
En cualquier caso, la norma no genera renta del suelo: solo permite concretar un aprovechamiento económico. De nuevo, las presiones de demanda por localización son las que generan renta del suelo en virtud de la necesaria asignación de quien ocupa un espacio que es limitado. Los ejemplos que soportan esta afirmación son múltiples. La ocupación de usos no permitidos en las ciudades y los enormes cambios de precios en algunas zonas de la ciudad indican que, aun sin cambio normativo o con el mantenimiento de la norma en esas zonas, los precios del suelo suben.

Cuando la administración de la ciudad expide la norma, por ejemplo, para cambiar el uso del suelo en zonas en las que se ha incrementado el uso comercial de forma irregular, o para incrementar la edificabilidad en una zona con altos precios del suelo, acepta que no entiende las dinámicas sociales y, en consecuencia, no puede dirigir las fuerzas del mercado hacia otros espacios urbanos, reconociendo así su incapacidad de control urbano. En el caso de Bogotá, el mercado ha mostrado demasiado poder en gran parte del territorio. Los hechos registrados señalan la preeminencia de esas fuerzas y, en los modelos, la irrelevancia de la norma en la dinámica del mercado.

La dinámica del comportamiento de los precios del suelo en dos zonas contiguas de usos comerciales y residenciales de alto ingreso, los propios gradientes expuestos por los precios del suelo de las zonas de la Lonja de Propiedad Raíz de Bogotá, sugieren un proceso de configuración urbana dominado completamente por el mercado, como lo muestran claramente los postulados de la teoría de la Renta Ofertada. Es evidente que se necesita evaluar qué opciones generan mayor bienestar, mercados autorregulados o el control urbano.

Finalmente, se necesita indagar sobre la importancia de los agentes urbanos y el poder tras las decisiones normativas en estas ciudades, mientras la no neutralidad de la norma debe ser investigada en sus efectos económicos sobre nuestras ciudades. En primer lugar, no hacer o dejar de hacer tiene un efecto de individualización de la riqueza colectiva. La indiferencia de la norma en el precio beneficia a los propietarios del suelo. En segundo lugar, si las demandas por localización y las ofertas de renta de los individuos son exógenas a la producción de espacio edificado, un propietario del suelo no agrega valor, solo lo apropia, indicando que un ajuste de este divorcio entre norma y sociedad se puede alcanzar con la captura de valor.

\section{Referencias bibliográficas}

Alburquerque, F. (2006). Clusters, territorio y desarrollo empresarial: Diferentes modelos de organización productiva. Fondo Multilateral de Inversiones (FOMIN) y Banco Interamericano de Desarrollo. https://publications.iadb.org/es/publicacion/14444/ clusters-territorio-y-desarrollo-empresarial-diferentes-modelos-de-organizacion

Alonso, W. (1964). Location and land use. Toward a general theory of land rent. Harvard University Press. https://doi.org/10.4159/harvard.9780674730854 
Amézquita, L., Rodríguez, L. \& Murillo, H. (2015). Los precios del suelo en Bogotá. El barrio Veinte de Julio. Bitácora Urbano Territorial, 25(1), 43-50. https://doi.org/10.15446/ bitacora.v $\ln 25.40236$

Aparicio, V., Avendaño, L. K., González, G. \& Peña, D. (2018). Análisis geoespacial de la dinámica de los humedales Meandro del Say y Santa María del Lago: Conflictos de usos del suelo y su pérdida de área. Revista Avances, 15(1), 131-149. https://doi.org/ https://doi.org/10.18041/1794-4953/avances.1.1374

Boisier, S. (2009). El retorno del actor territorial a su nuevo escenario. Ambienta, (89), 112141. https://dialnet.unirioja.es/servlet/articulo?codigo $=3275010$

Brower, S. N. (1980). Territory in urban settings. En I. Altman, A. Rapoport \& J. F. Wohlwill (Eds.), Environment and culture. Human behavior and environment (Advances in theory and research). Vol. 4. Springer. https://doi.org/10.1007/978-1-4899-0451-5_6

Brueckner, J. K. (2011). Lectures on Urban Economics. Cambridge, Massachusetts: The MIT Press.

Burgess, E. W. (1925). The growth of the city. En R. E. Park, E. W. Burgess \& R. D. McKenzie (Eds.), The city. The University of Chicago Press.

Camargo, A. \& Hurtado, A. (2013). Urbanización informal en Bogotá: agentes y lógicas de producción del espacio urbano. Revista INVI, 28(78), 77-107. https://doi.org/10.4067/ S0718-83582013000200003

Chapin, F. S. \& Hightower, H. C. (1965). Household activity patterns and land use. Journal of the American Planning Association, 31(3), 222-231. https://doi. org $/ 10.1080 / 01944366508978169$

Cruz, D., Motta, J. \& García, C. A. (2017). Estimación de la pérdida de área en los humadelas de Bogotá en las últimas cinco decadas debido a la construcción y sus respectivos efectos. Documento de Trabajo, Universidad Distrital Francisco José de Caldas. http:// repository.udistrital.edu.co/bitstream/11349/5345/1/CruzSolanoDianaPaola2017.pdf

Cuervo-González, L. M. (1998). Desarrollo económico local: leyendas y realidades. Territorios, (1), 9-24. https://revistas.urosario.edu.co/index.php/territorios/article/view/5605

Dear, M. J. \& Flusty, S. (Eds.). (2002). The spaces of postmodernity readings in human geography. Blackwell.

Delgado, J. \& Galindo, C. (2006). Los espacios emergentes de la dinámica rural-urbana. Problemas Del Desarrollo. Revista Latinoamericana de Economía, 37(147), 187-216. https://doi.org/10.22201/iiec.20078951e.2006.147.7639

Departamento Administrativo Nacional de Estadísticas (DANE), Colombia. (2019). Resultados Censo Nacional de Población y Vivienda 2018. https://www.dane.gov.co/files/ censo2018/informacion-tecnica/cnpv-2018-presentacion-3ra-entrega.pdf

Enders, W. (1995). Applied econometric time series analysis. Wiley and Sons.

Fernandes, E. (2011). Regularización de asentamientos informales en América Latina. Informe sobre Enfoque en Politicas de Suelo. Lincoln Institute of Land Policy. https://www. lincolninst.edu/sites/default/files/pubfiles/regularizacion-asentamientos-informalesfull_0.pdf

Fischel, W. A. (2000). Zoning and land use regulation. En B. Boudewijn \& G. D. Geest (Eds.), Encyclopedia of Law and Economics (pp. 403-442). Vol. 2. Edward Elgar. http://www. dartmouth.edu/ -wfischel/Papers/WAF-zoning ELEpdf.pdf

Fujita, M. (1989). Urban economic theory. Land use and city size. Cambridge University Press. 
Fujita, M. (2010). The evolution of spatial economics: From Thünen to the New Economic Geography. The Japanese Economic Review, 61(1), 1-32. https://doi.org/10.1111/ j.1468-5876.2009.00504.x

Fujita, M. \& Krugman, P. (1995). When is the economy monocentric?: von Thünen and Chamberlin unified. Regional Science and Urban Economics, 25(4), 505-528. https:// doi.org/10.1016/0166-0462(95)02098-F

Fujita, M. \& Thisse, J. F. (2013). Economics of agglomeration: Cities, industrial location, and globalization ( $2^{\text {nd }}$ ed.). Cambridge University Press.

Fundación Demuca [Fundación para el Desarrollo Local y el Fortalecimiento Municipal e Institucional de Centroamérica y el Caribe]. (2009). Guia de herramientas municipales para la promoción del desarrollo económico local. https://www.cepal.org/sites/default/ files/guia_herramientas_municipales_demuca.pdf

Glaeser, E. L. \& Gyourko, J. (2002). The impact of zoning on housing affordability. NBER (Working Paper 8835). https://doi.org/10.3386/w8835

Harris, C. D. \& Ullman, E. L. (1945). The nature of cities. The Annals of the American Academy of Political and Social Science, 242(1), 7-17. https://doi. org/10.1177/000271624524200103

Helsley, R. W. \& Sullivan, A. M. (1991). Urban subcenter formation. Regional Science and Urban Economics, 21(2), 255-275. https://doi.org/10.1016/0166-0462(91)90036-M

Henderson, V. \& Becker, R. (2000). Political economy of city sizes and formation. Journal of Urban Economics, 48(3), 453-484. https://doi.org/10.1006/juec.2000.2176

Hoyt, H. (1933). One hundred years of land values in Chicago. The University of Chicago Press. https://archive.org/details/onehundredyearso00hoytrich

Huxley, M. (1997). "Necessary but by no means sufficient..." Spatial political economy, town planning and the possibility of better cities: A commentary on Brian McLoughlin's last paper. European Planning Studies, 5(6), 741-751. https://doi. org/10.1080/09654319708720432

IDOM (Consulting, Engineering, Architecture S.A.u.), Secretaría Distrital de Planeación (SDP), Gobernación de Cundinamarca \& Findeter. (2018). Análisis histórico y evolución de la huella urbana. En Estudio de crecimiento y evolución de la huella urbana para los municipios que conforman el área Bogotá región (Cap. 2). http://www.sdp.gov.co/ sites/default/files/diagnostico_de_la_huella_urbana_de_bogota_y_20_municipios_ de_1997_a_2016.pdf

Jacobs, J. (1969). The economy of cities. Vintage.

Jaramillo, S. (2009). Hacia una teoría de la renta del suelo urbano (2a ed.). Universidad de los Andes, Facultad de Economía, Centro de Estudios sobre Desarrollo Económico (CEDE), Ediciones Uniandes.

Lonja de Propiedad Raíz de Bogotá (LPRB). (2017). Estudio del valor del suelo urbano en Bogotá 2014-2016

Luengo, G. (2002). La calidad ambiental urbana como instrumento teórico-metodológico. Estudio del impacto sobre valores histórico-urbanísticos. Fermentum, 12(33), 126141. https://www.redalyc.org/pdf/705/70511244009.pdf

Lütkepohl, H. \& Krätzig, M. (2004). Applied Time Series Econometrics. Cambridge University Press. 
Madsen, H. (2007). Time Series Analysis. Chapman Hall. https://doi.org/10.1201 19781420059687

Martínez, E. (2014). Configuracion urbana, hábitat y apropiación del espacio. XIII Coloquio Internacional de Geocritica. El control del espacio y los espacios de control. Barcelona, 5-10 de mayo de 2014. http://www.ub.edu/geocrit/coloquio2014/Emilio\%20Martinez.pdf

Maser, S. M., Riker, W. H. \& Rosett, R. N. (1977). Effects of zoning and externalities on the prices of land in Monroe County, New York (USA). The Journal of Law and Economics, 20(1), 111-132. https://doi.org/http://dx.doi.org/10.1086/466894

McLoughlin, J. B. (1994). Centre or periphery? Town planning and spatial political economy. Environment and Planning A: Economy and Space, 26(7), 1111-1122. https://oi. org/10.1068/a261111

Monk, S., Pearce, B. J. \& Whitehead, C. M. E. (1996). Land-use planning, land supply, and house prices. Environment and Planning A, 28(3), 495-511. https://doi.org/10.1068/ a280495

Naclerio, A., Roura, H. \& Giorgi, D. (2010). Sistemas productivos locales, politicas públicas y desarrollo económico. Programa Naciones Unidas para el Desarrollo (PNUD). https:// www.undp.org/content/dam/argentina/Publications/DesarrolloInclusivo/Sistemas Productivos Locales.pdf

Pollakowski, H. O. \& Wachter, S. M. (1990). The effects of land-use constraints on housing prices. Land Economics, 66(3), 315-324. https://doi.org/10.2307/3146732

Salazar, A. \& Vergel, E. (2009). Control Urbano en Aglomeraciones Urbanas. Seminario viii de Investigación Urbano Regional - aciur, Gobierno de Municipios y Aglomeraciones Urbanas. http://www.institutodeestudiosurbanos.info/eventos/seminarios-de-investi gacion-urbano-regional-aciur/memorias-viii-seminario-aciur-2009/mesas-tematicas/ fragmentacion-apropiacion-y-regulacion/253-control-urbano-en-aglomeracionesurbanas/file

Server, O. B. (1996). Corruption: A major problem for urban management: Some evidence from Indonesia. Habitat International, 20(1), 23-41. https://doi.org/10.1016/01973975(95)00032-1

Thorson, J. A. (1994). Zoning policy changes and the urban fringe land market. Real Estate Economics, 22(3), 527-538. https://doi.org/10.1111/1540-6229.00647

Thorson, J. A. (1997). The effect of zoning on housing construction. Journal of Housing Economics, 6(1), 8191. https://doi.org/10.1006/jhec.1997.0205

Vigliocco, M. A. \& Meda, R. H. (1991). Estructura urbana y uso del suelo. Civilidad. https:// es.scribd.com/document/178451202/Estructura-Urbana-y-Uso-Del-Suelo-ViglioccoMeda

Wallace, N. E. (1988). The market effects of zoning undeveloped land: Does zoning follow the market? Journal of Urban Economics, 23(3), 307-326. https://doi.org/10.1016/00941190(88)90021-6

Yiftachel, O. (1997). Too little, but not too late: Planning theory and the work of Brian McLoughlin. European Planning Studies, 5(6), 765-770. https://doi. org/10.1080/09654319708720435

Yunda, J. G. (2019). Densificación y estratificación social en Bogotá: Distribución sesgada de la inversión privada. EURE, 45(134), 237-257. https://doi.org/10.4067/S025071612019000100237 